\title{
Calretinin Expression in Unicystic Ameloblastoma and Odontogenic Cysts
}

\author{
Efetobo Victor Orikpete ${ }^{*}$, Osawe Felix Omoregie ${ }^{2}$, Michael Akin 0jo² \\ ${ }^{1}$ University of Port Harcourt, Port Harcourt, Nigeria \\ ${ }^{2}$ University of Benin, Benin, Nigeria \\ Email: ${ }^{*}$ efezi2000@yahoo.com
}

How to cite this paper: Orikpete, E.V., Omoregie, O.F. and Ojo, M.A. (2020) Calretinin Expression in Unicystic Ameloblastoma and Odontogenic Cysts. Journal of Biosciences and Medicines, 8, 111-120. https://doi.org/10.4236/jbm.2020.84010

Received: February 13, 2020

Accepted: April 14, 2020

Published: April 17, 2020

Copyright $\odot 2020$ by author(s) and Scientific Research Publishing Inc. This work is licensed under the Creative Commons Attribution International License (CC BY 4.0).

http://creativecommons.org/licenses/by/4.0/

(c) (i) Open Access

\begin{abstract}
Background: Unicystic ameloblastoma arises from similar tissues as the odontogenic cysts. It may also arise from the epithelial lining of these cysts. Furthermore, the epithelial lining of unicystic ameloblastoma is variable and may be almost completely nondescript, which may lead to diagnostic confusion with odontogenic cysts. Calretinin is a calcium-binding protein that has been suggested to be a specific immunohistochemical marker for ameloblastic tissues. The aim of this study was to investigate the usefulness of calretinin in the differentiating unicystic ameloblastoma from odontogenic keratocyst, dentigerous cyst and radicular cyst of the jaws. Materials and Methods: Formalin-fixed, paraffin embedded tissue blocks of 23 unicystic ameloblastomas, 6 odontogenic keratocysts, 8 dentigerous cysts and 10 radicular cysts were utilized. Five micrometer thick sections were made from the tissue blocks and mounted on silanized glass slides. Immunohistochemical staining was done using a 1:100 dilution of mouse monoclonal anti-calretinin primary antibodies (Abcam, SA; Clone 1F5H1) and incubating overnight at $4^{\circ} \mathrm{C}$. The staining reaction was visualised using diaminobenzidine substrate solution. Stained tissue sections were evaluated for the presence, distribution, and intensity of staining of the immunoreactive cells. Data analysis was done using SPSS version 23. Statistical tests employed were Chi square test and Kruskal-Wallis test. The level of significance was set at $95 \%$ ( $p$-value $<0.05)$. Results: Calretinin expression was seen in 14 (60.9\%) cases of unicystic ameloblastoma, $3(50.0 \%)$ cases of odontogenic keratocyst and a lone (12.5\%) case of dentigerous cyst. None of the 10 cases of radicular cyst showed positive expression of calretinin. Staining in unicystic ameloblastoma was mostly localized to the superficial layers, whereas in odontogenic keratocyst it involved all layers of the epithelial lining. There was significant association between lesion type and the immunohistochemical expression of calretinin $(p=0.003)$. There were also significant differences in calretinin immunoreactivity of un-
\end{abstract}


icystic ameloblastoma compared to dentigerous cyst and radicular cyst. Conclusion: The findings of this study suggest that calretinin expression could help differentiate unicystic ameloblastoma from dentigerous cyst and radicular cyst, but not from odontogenic keratocyst.

\section{Keywords}

Calretinin, Odontogenic, Cyst, Ameloblastoma

\section{Introduction}

Ameloblastoma is a benign odontogenic tumour of epithelial origin and is the most common odontogenic tumour in Africa [1] [2]. It arises from epithelial remnants of the dental lamina, enamel organ, reduced enamel epithelium, epithelial cell rests of Malassez, basal layer of the oral mucosa, as well as the epithelial lining of odontogenic cysts like dentigerous cyst, radicular cyst and odontogenic keratocyst (keratocystic odontogenic tumour) [3] [4]. Unicystic ameloblastoma (UA) is a clinico-pathologically distinct type of ameloblastoma comprising $5 \%$ to $22 \%$ of all reported cases of intraosseous ameloblastoma [5] [6]. It displays clinical, radiographic or gross features of a jaw cyst, but shows an epithelial lining composed focally or entirely of ameloblastomatous epithelium on histopathologic examination [5] [6].

Odontogenic cysts are cysts that develop from tooth-forming epithelium. Common examples include odontogenic keratocyst (OKC), dentigerous cyst (DC) and radicular cyst (RC) [7] [8]. Odontogenic keratocyst arises from the dental lamina and its remnants [9]. Dentigerous cyst develops from the reduced enamel epithelium, while radicular cyst has its origin from the epithelial cell rests of Malassez [9]. Odontogenic keratocyst was classified by the WHO in 2005 as an odontogenic tumour, because of its potential for aggressive behaviour and a high recurrence rate. The WHO however in 2017 re-classified it as an odontogenic cyst [10].

Owing to the similar tissue(s) of origin of unicystic ameloblastoma, odontogenic keratocyst, dentigerous cyst and radicular cyst, compounded by the fact that unicystic ameloblastoma may arise from the epithelial lining of these lesions accurate differential diagnosis is sometimes challenging [11]. Furthermore, the epithelial lining of unicystic ameloblastoma is variable and may sometimes be almost completely nondescript, which may lead to diagnostic confusion with odontogenic cysts [12] [13]. The entire epithelial lining of UA may show this ameloblastomatous change, but more often this is restricted to only a focal area [12] [13]. In fact, substantial portions of the cyst may be lined by a non-specific epithelium, bearing similarity to the lining epithelium of other odontogenic cysts of the jaws such as odontogenic keratocyst, dentigerous cyst and radicular cyst [14] [15]. In such cases, histological diagnosis can be difficult, and examination 
of only a portion of the cyst lining may lead to a misdiagnosis.

Several immunohistochemical markers, including calretinin, have been used in an attempt to differentiate unicystic ameloblastoma from odontogenic cysts. Calretinin is a calcium-binding protein of 29-kilodalton $(29-\mathrm{kD})$ and is a member of the large family of EF-hand proteins to which the S100 protein also belongs [16]. It is widely expressed in central and peripheral neural tissues particularly in the retina and in neurones of sensory pathways [17], although it is also expressed in non-neural tissues [18]. Its expression has been described in neural elements of the pulp and periodontal ligament, as well as in the odontogenic epithelium during the process of odontogenesis [19] [20]. Its exact biological role remains unknown, though possible roles as a calcium buffer and/or calcium sensor and regulator of apoptosis have been postulated [21]. Calretinin has been suggested to be a specific immunohistochemical marker for ameloblastic tissues, and an important diagnostic aid in the differential diagnosis of cystic odontogenic lesions and ameloblastic tumours [22].

A number of studies have investigated the role of calretinin in differentiating ameloblastoma from other odontogenic tumours [23] [24]. Few studies have reported the expression of calretinin in unicystic ameloblastoma and odontogenic cysts. This study aimed to investigate the usefulness of calretinin in the differentiating unicystic ameloblastoma from odontogenic keratocyst, dentigerous cyst and radicular cyst of the jaws.

\section{Materials and Methods}

\subsection{Samples}

This study was carried out at the Department of Oral Pathology/Medicine, University of Benin Teaching Hospital between January 2016 and December 2016. Formalin-fixed, paraffin embedded tissue blocks of twenty-three (23) unicystic ameloblastomas, 6 odontogenic keratocysts, 8 dentigerous cysts and 10 radicular cysts were selected from the archival specimens of the Department of Oral Pathology/Medicine, University of Benin Teaching Hospital. Confirmation of the previous diagnosis was done by taking fresh sections from the tissue blocks for staining with haematoxylin and eosin. Human brain tissue was used as positive control.

\subsection{Immunohistochemistry}

Five micrometer thick sections were made from the tissue blocks and mounted on silanized glass slides. Specimens were deparaffinised in xylene and hydrated by passing through descending grades of alcohol. Antigen retrieval was done by immersing in citrate buffer and incubating at $95^{\circ} \mathrm{C}$ in a water bath for $20 \mathrm{mi}$ nutes.

The tissue area on the slides was marked with a hydrophobic pen. Endogenous peroxidase activity was blocked by applying $3 \%$ hydrogen peroxide solution in methanol (Dako, Denmark) and incubating at room temperature for $20 \mathrm{mi}$ - 
nutes, following which a serum-free protein block was added and slides incubated at room temperature for 5 minutes to prevent non-specific reactions.

A 1:100 dilution of mouse monoclonal anti-calretinin primary antibodies (Abcam, SA; Clone 1F5H1) was applied and incubation was done overnight at $4^{\circ} \mathrm{C}$. Appropriately diluted horseradish peroxidase polymer detection solution (Dako, Denmark) was then applied, and the slides incubated in a humidified chamber at room temperature for 15 minutes, following which diaminobenzidine (DAB) substrate solution (Dako, Denmark) was added to reveal the colour of antibody reaction. Counterstaining was done by immersing slides in Gill's haematoxylin for 20 seconds. The slides were then rinsed in distilled water, dehydrated in ascending grades of alcohol, cleared in xylene and cover slipped using distyrene plasticizer in xylene (DPX) as mountant.

\subsection{Interpretation of Staining}

Stained tissue sections were evaluated for the presence, distribution, and intensity of staining of the immunoreactive cells [12] [23]. Positive staining was seen as a brownish colouration involving both the nucleus and cytoplasm. Distribution was determined as either being focal (involving $<50 \%$ of the epithelium) or diffuse (involving $>50 \%$ of the epithelium). The intensity was graded as follows: $0=$ no staining; $(+)=$ weak staining; $(++)=$ moderate staining; and $(+++)=$ in tense staining.

\subsection{Statistical Analysis}

Data analysis was done using the Statistical Package for Social Sciences (SPSS) version 23 software (IBM Corp., 2015). Chi square statistical test was employed to determine association between lesion type and immunohistochemical expression of markers. A non-parametric ANOVA was used to determine significant differences in the immunoreactivity of calretinin, and pairwise comparison was with the aid of Kruskal-Wallis test. The level of significance was set at 95\% ( $p$-value $<0.05)$.

\section{Results}

Unicystic ameloblastomas showed positive immunohistochemical expression of calretinin in 14 (60.9\%) cases, most of which were diffuse $(n=8,57.1 \%)$. Equal number of cases showed mild $(n=6,42.9 \%)$ and moderate $(n=6,42.9 \%)$ staining intensity. Staining was mostly localized to the stellate reticulum-like cells in the superficial layers of the epithelial lining and the central areas of tumour islands (Figure 1). Odontogenic keratocyst showed positive calretinin expression in $3(50.0 \%)$ cases. The distribution was diffuse in $2(66.7 \%)$ of the 3 positive cases. The staining involved all layers of the epithelial lining (Figure 2). One case $(12.5 \%)$ of dentigerous cyst was positive for calretinin. The reaction was focal and of moderate intensity (Figure 3 ). None of the 10 cases of radicular cyst showed positive expression of calretinin (Figure 4) [Table 1]. 


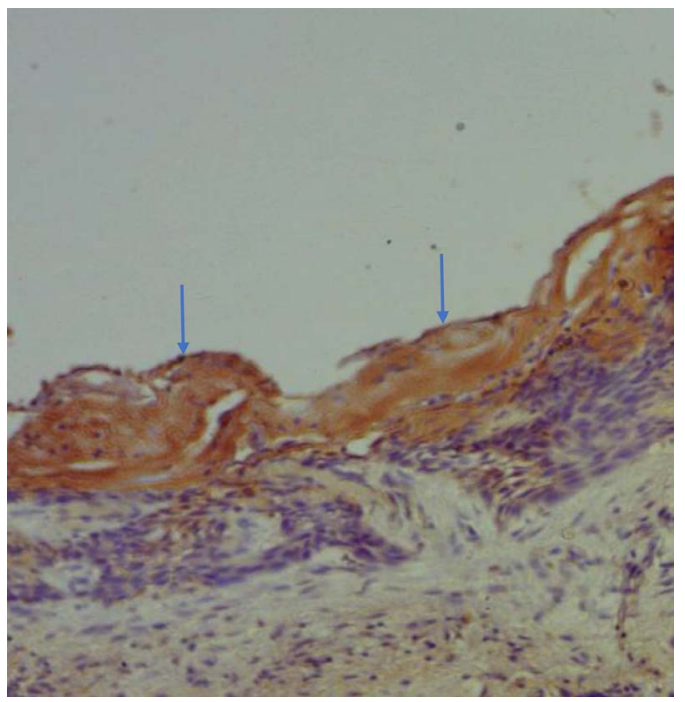

Figure 1. Photomicrograph of unicystic ameloblastoma showing intense expression of calretinin, mainly in the superficial layer $[\times 400]$.

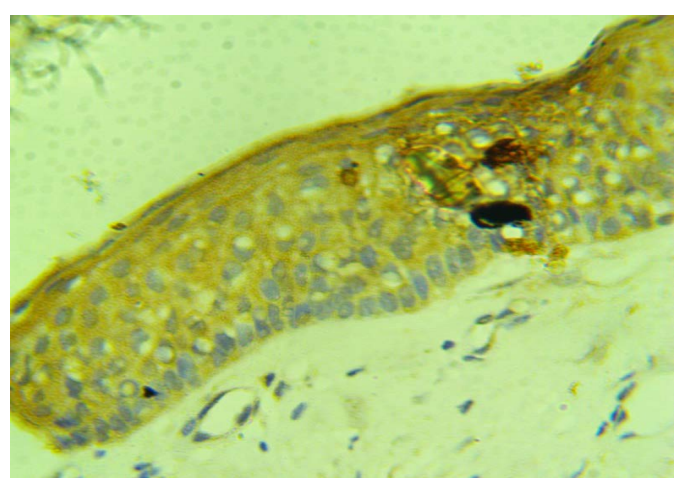

Figure 2. Photomicrograph of odontogenic keratocyst showing diffuse expression of calretinin involving all layers of the epithelial lining $[\times 400]$.

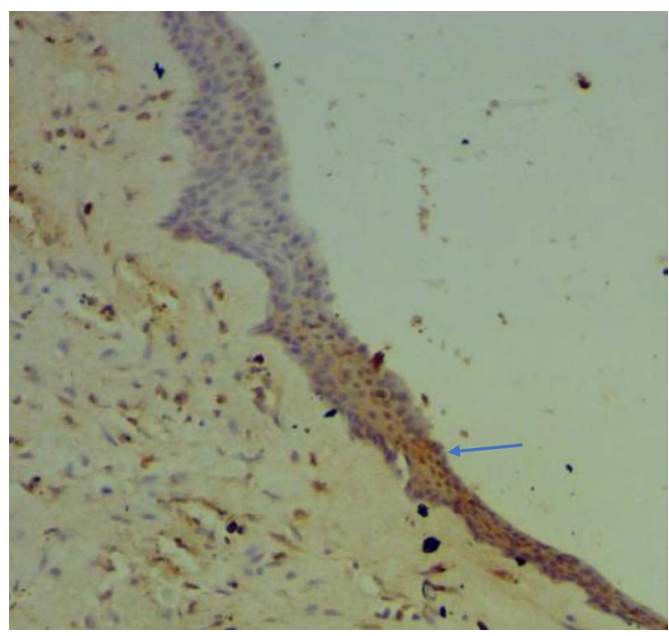

Figure 3. Dentigerous cyst showing focal expression of calretinin $[\times 100]$. 


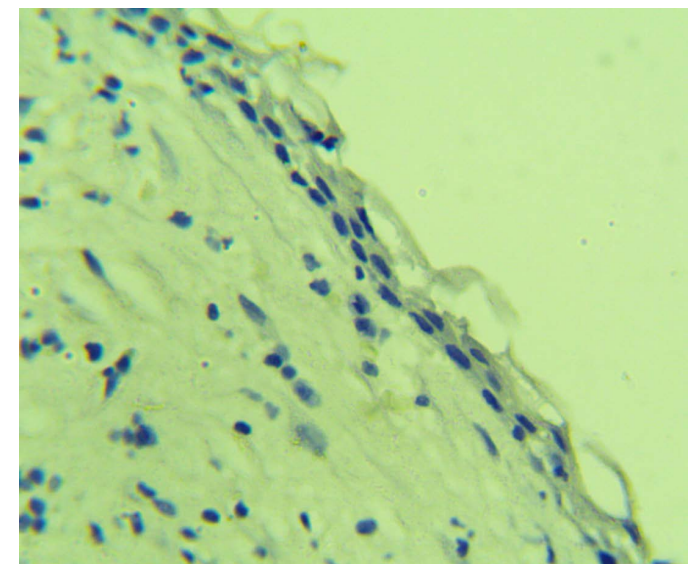

Figure 4. Radicular cyst showing negative calretinin expression $[\times 400]$.

Table 1. Calretinin expression (frequency, intensity and distribution) in the patients.

\begin{tabular}{ccccccccc}
\hline \multirow{2}{*}{$\begin{array}{c}\text { Histologic } \\
\text { diagnosis }\end{array}$} & Total & $\begin{array}{c}\text { Calretinin } \\
\text { cases }\end{array}$ & \multicolumn{2}{c}{$\begin{array}{c}\text { Intensity of Calretinin } \\
\text { expession } \\
\text { c\%) }\end{array}$} & & \multicolumn{2}{c}{$\begin{array}{c}\text { Distribution } \\
\text { of calretinin } \\
\text { expression (\%) }\end{array}$} \\
\cline { 5 - 9 } & & & Mild & Moderate & Intense & Focal & Diffuse \\
\hline UA & 23 & $14(60.9)$ & $6(42.9)$ & $6(42.9)$ & $2(14.3)$ & $6(42.9)$ & $8(57.1)$ \\
OKC & 6 & $3(50.0)$ & $1(33.3)$ & $1(33.3)$ & $1(33.3)$ & $1(33.3)$ & $2(66.7)$ \\
DC & 8 & $1(12.5)$ & - & $1(100.0)$ & - & $1(100.0)$ & - \\
RC & 10 & $0(0.0)$ & - & - & - & - & - \\
\hline
\end{tabular}

Key: UA $=$ Unicystic ameloblastoma, $\mathrm{OKC}=$ Odontogenic keratocyst, $\mathrm{DC}=$ Dentigerous cyst, $\mathrm{RC}=$ Radicular cyst.

There was significant association between lesion type and the immunohistochemical expression of calretinin $(p=0.003)$. A non-parametric ANOVA (Kruskal-Wallis) test showed significant differences in the immunoreactivity of calretinin across the categories of lesions studied. $(p=0.004)$ A pairwise post-hoc analysis showed significant differences in calretinin immunoreactivity of unicystic ameloblastoma compared to dentigerous cyst and radicular cyst. Likewise, there was significant difference between the calretinin immunoreactivity between keratocystic odontogenic tumour and radicular cyst (Table 2).

\section{Discussion}

Unicystic ameloblastoma is a benign odontogenic tumour with clinicoradiologic features that are similar to those of other non-neoplastic jaw cysts [25]. Radicular cyst, dentigerous cyst, and odontogenic keratocyst are common odontogenic cysts of the jaws, with considerable overlap of their diagnostic histopathologic features, especially in the presence of an on-going inflammatory reaction [11] [26]. Immunohistochemical expression of calretinin was evaluated in this study to differentiate these lesions with overlapping diagnostic clinicopathologic features. 
Table 2. Pairwise comparison (Kruskal-Wallis test) of calretinin immunoreactivity among the lesions studied.

\begin{tabular}{cccc}
\hline Sample 1 - Sample 2 & Standard Error & Significance & $\boldsymbol{p}=\mathbf{0 . 0 0 4}$ \\
\hline RC - DC & 5.477 & 0.592 & \\
RC - KCOT & 5.963 & $\mathbf{0 . 0 4 9}$ & \\
RC - UA & 4.374 & $\mathbf{0 . 0 0 1}$ \\
DC - KCOT & 6.236 & 0.158 \\
DC - UA & 4.740 & $\mathbf{0 . 0 1 6}$ \\
KCOT - UA & 5.293 & 0.629 \\
\hline
\end{tabular}

Key: $\mathrm{UA}=$ Unicystic ameloblastoma, $\mathrm{KCOT}=$ Keratocystic odontogenic tumour, DC $=$ Dentigerous cyst, $\mathrm{RC}=$ Radicular cyst.

Expression of calretinin in unicystic ameloblastoma in this study was seen in $14(60.9 \%)$ cases. This is relatively higher compared to the 50\% recorded in 16 cases of unicystic ameloblastomas by Anandani et al. [12]. It is however lower than the values reported by Pawar et al. [27] and Altini et al. [22], who reported positive expression of calretinin in $80 \%$ and $81.5 \%$ respectively of unicystic ameloblastomas. Furthermore, DeVilliers et al. [18], Rudraraju et al. [28] and Sundaragiri et al. [15] reported positive expression of calretinin in $100 \%$ of unicystic ameloblastomas. Other studies have also found a 100\% expression of calretinin in ameloblastomas [20] [22] [23] [29] [30]. This study recorded localization of calretinin immunoreaction to the stellate reticulum-like cells and the superficial layers of the epithelial lining. This is similar to the findings reported by other researchers [12] [20] [22] [24] [31].

This study also recorded positive immunohistochemical expression of calretinin in 3 (50.0\%) cases of OKC. Whereas, Anandani et al. [12] and Imran et al. [30] reported positive calretinin expression in $5 \%$ and $13 \%$ of OKCs respectively. Some studies have found higher values for the expression of calretinin in OKC ranging from $40 \%$ to $91.7 \%$ [20] [24] [27] [32]. D'Silva et al. [20] reported $40 \%$ expression, Piatelli et al., [32] reported 66.7\% expression; Koneru et al. [24] reported $80 \%$ expression, while Pawar et al. [27] reported 91.7\% expression. Other authors have however reported no expression of calretinin in OKCs [15] [18] [29] [31]. In this study, the staining reaction for calretinin in OKC involved all layers of the epithelial lining. A similar finding was reported by Koneru et al. [24] in $92 \%$ of OKC that showed positive calretinin expression. Other reports however found calretinin expression in OKC localized to the suprabasal and intermediate/superficial layers of the epithelium [27] [32].

There was positive immunohistochemical expression of calretinin in $1(12.5 \%)$ of the cases of dentigerous cyst in this study. This is in contrast to the $0 \% \mathrm{im}$ munoreactivity to calretinin antibody reported by several authors [15] [20] [29] [30] [31]. The lone case found in this study may represent early ameloblastomatous change in the lining epithelium of dentigerous cyst. There was no (0\%) expression of calretinin in all the cases of radicular cyst studied. Similar findings 
have been reported in several studies [20] [30] [31] [32].

This study observed significant differences in the calretinin expression/ immunoreactivity between unicystic ameloblastoma, and those of dentigerous cyst and radicular cyst. Previous reports in the literature corroborate this finding [15] [20] [31]. Also, there was a significant difference in calretinin immunoreactivity between odontogenic keratocyst and radicular cyst. These findings suggest that calretinin may help in differentiating unicystic ameloblastoma from dentigerous cyst and radicular cyst, as well as differentiating odontogenic keratocyst from radicular cyst. There was no significant difference in the calretinin immunoreactivity between unicystic ameloblastoma and odontogenic keratocystin this study, which is similar to the findings of Koneru et al. [24]. However, there was a difference in the localization of the immunoreaction. Whereas unicystic ameloblastoma showed immunoreactivity to calretinin mainly in the superficial layers of the lining epithelium, odontogenic keratocyst involved all layers of the epithelial lining. Further studies are encouraged to elucidate the reason for this differential pattern of localization of the immunoreaction of calretinin, to ascertain if it can help differentiate between the two lesions.

\section{Conclusion}

The findings of this study suggest that calretinin expression could help differentiate unicystic ameloblastoma from dentigerous cyst and radicular cyst. However, calretinin expression could not reliably differentiate unicystic ameloblastoma from odontogenic keratocyst. Further studies employing other advanced diagnostic techniques such as polymerase chain reaction as well as genetic studies are encouraged to shed further light on the expression and role of calretinin in the aetiopathogenesis of ameloblastoma and odontogenic keratocyst in particular.

\section{Conflicts of Interest}

The authors declare no conflicts of interest regarding the publication of this paper.

\section{References}

[1] Ladeinde, A.L., Ogunlewe, M.O., Bamgbose, B.O., Adeyemo, W.L., Ajayi, O.F., Arotiba, G.T., et al. (2006) Ameloblastoma: Analysis of 207 Cases in a Nigerian Teaching Hospital. Quintessence International, 37, 69-74.

[2] Lawal, A.O., Adisa, A.O. and Olusanya, A.A. (2013) Odontogenic Tumours: A Review of 266 Cases. Journal of Clinical and Experimental Dentistry, 5, e13-e17. https://doi.org/10.4317/jced.50949

[3] Omoregie, F.O., Sede, M.A. and Ojo, A.M. (2015) Ameloblastomatous Change in Radicular Cyst of the Jaw in a Nigerian Population. Ghana Medical Journal, 49, 107-111. https://doi.org/10.4314/gmj.v49i2.8

[4] Bhushan, N.S., Rao, N.M., Navatha, M. and Kumar, B.K. (2014) Ameloblastoma Arising from a Dentigerous Cyst: A Case Report. Journal of Clinical and Diagnostic Research, 8, ZD23-ZD25. 
[5] Sah, P., Menon, A., Kamath, A., Chandrashekar, C., Carnelio, S. and Radhakrishnan, R. (2013) Role of Immunomarkers in the Clinicopathological Analysis of Unicystic Ameloblastoma. Disease Markers, 35, 481-488.

https://doi.org/10.1155/2013/517834

[6] Philipsen, H.P. and Reichart, P.A. (1998) Unicystic Ameloblastoma: A Review of 193 Cases from the Literature. Oral Oncology, 34, 317-325. https://doi.org/10.1016/S1368-8375(98)00012-8

[7] Meningaud, J., Oprean, N., Pitak-Arnnop, P. and Bertrand, J. (2006) Odontogenic Cysts: A Clinical Study of 695 Cases. Journal of Oral Science, 48, 59-62. https://doi.org/10.2334/josnusd.48.59

[8] Acikgoz, A., Uzun-Bulut, E., Ozden, B. and Gunduz, K. (2012) Prevalence and Distribution of Odontogenic and Nonodontogenic Cysts in a Turkish Population. $\mathrm{Me}$ dicina Oral, Patologia Oral y Cirugia Bucal, 17, e108-e115. https://doi.org/10.4317/medoral.17088

[9] Shear, M. and Speight, P.M. (2007) Cysts of the Oral and Maxillofacial Regions. 4th Edition, Blackwell Munksgaard, Oxford, UK. https://doi.org/10.1002/9780470759769

[10] El-Naggar, A.K., Chan, J.K.C., Grandis, J.R., Takata, T. and Slootweg, P.J. (2017) Odontogenic and Maxillofacial Bone Tumours. In: WHO Classification of Head and Neck Tumours, 4th Edition, IARC, Lyon.

[11] Barrett, A.W., Sneddon, K.J., Tighe, J.V., Gulati, A., Newman, L., Collyer, J., et al. (2017) Dentigerous Cyst and Ameloblastoma of the Jaws: Correlating the Histopathological and Clinicoradiological Features Avoids a Diagnostic Pitfall. International Journal of Surgical Pathology, 25, 141-147. https://doi.org/10.1177/1066896916666319

[12] Anandani, C., Metgud, R. and Singh, K. (2014) Calretinin as a Diagnostic Adjunct for Ameloblastoma. Pathology Research International, 2014, Article ID: 308240. https://doi.org/10.1155/2014/308240

[13] Savithri, V., Janardhanan, M. and Rakesh, S. (2014) Unicystic Ameloblastoma as a Differential Diagnosis for Odontogenic Cysts. Oral and Maxillofacial Pathology Journal, 5, 466-469.

[14] Li, T.J. (2007) The Unicystic Ameloblastoma: A Clinicopathologically Distinct Entity. Chinese Journal of Dental Research, 10, 7-13.

[15] Sundaragiri, S.K., Chawda, J., Gill, S., Odedra, S. and Parmar, G. (2010) Calretinin Expression in Unicystic Ameloblastoma: An Aid in Differential Diagnosis. The Journal of Oral Biosciences, 52, 164-169. https://doi.org/10.1016/S1349-0079(10)80046-5

[16] Rogers, J., Khan, M. and Ellis, J. (1990) Calretinin and Other CaBPs in the Nervous System. Advances in Experimental Medicine and Biology, 269, 195-203. https://doi.org/10.1007/978-1-4684-5754-4_32

[17] Schwaller, B., Buchwald, P., Blumcke, I., Celio, M.R. and Hunziker, W. (1993) Characterisation of a Polyclonal Antiserum against Purified Human Recombinant Calcium Binding Protein Calretinin. Cell Calcium, 14, 639-648. https://doi.org/10.1016/0143-4160(93)90089-O

[18] Devilliers, P., Liu, H., Suggs, C., Simmons, D., Daly, B., Zhang, S., et al. (2008) Calretinin Expression in the Differential Diagnosis of Human Ameloblastoma and Keratocystic Odontogenic Tumour. The American Journal of Surgical Pathology, 32, 256-260. https://doi.org/10.1097/PAS.0b013e3181452176

[19] Mistry, D., Altini, M., Coleman, H.G., Ali, H. and Maiorano, E. (2001) The Spatial 
and Temporal Expression of Calretinin in Developing Rat Molars (Rattus norvegicus). Archives of Oral Biology, 46, 973-981. https://doi.org/10.1016/S0003-9969(01)00046-2

[20] D’Silva, S., Sumathi, M.K., Balaji, N., Shetty, N.K., Pramod, K.M. and Cheeramelil, J. (2013) Evaluation of Calretinin Expression in Ameloblastoma and Non-Neoplastic Odontogenic Cysts-An Immunohistochemical Study. Journal of International Oral Health, 5, 42-48.

[21] Dei Tos, A.P. and Doglioni, C. (1998) Calretinin: A Novel Tool for Diagnostic Immunohistochemistry. Advances in Anatomic Pathology, 5, 61-66. https://doi.org/10.1097/00125480-199801000-00052

[22] Altini, M., Coleman, H., Doglioni, C., Favia, G. and Maiorano, E. (2000) Calretinin Expression in Ameloblastomas. Histopathology, 37, 27-32. https://doi.org/10.1046/j.1365-2559.2000.00940.x

[23] Alaeddini, M., Etemad-Moghadam, S. and Baghaii, F. (2008) Comparative Expression of Calretinin in Selected Odontogenic Tumours: A Possible Relationship to Histogenesis. Histopathology, 52, 299-304. https://doi.org/10.1111/j.1365-2559.2007.02948.x

[24] Koneru, A., Hallikeri, K., Nellithady, G.S., Krishnapillai, R. and Prabhu, S. (2014) Immunohistochemical Expression of Calretinin in Ameloblastoma, Adenomatoid Odontogenic Tumor, and Keratocystic Odontogenic Tumor: A Comparative Study. Applied Immunohistochemistry \& Molecular Morphology, 22, 762-767. https://doi.org/10.1097/PAI.0000000000000005

[25] Masthan, K., Anitha, N., Krupaa, J. and Manikkam, S. (2015) Ameloblastoma. Journal of Pharmacy and Bioallied Sciences, 7, S167-S170. https://doi.org/10.4103/0975-7406.155891

[26] Veena, K.M., Rao, R., Jagadishchandra, H. and Rao, P.K. (2011) Odontogenic Keratocyst Looks Can Be Deceptive, Causing Endodontic Misdiagnosis. Case Reports in Pathology, 2011, Article ID: 159501. https://doi.org/10.1155/2011/159501

[27] Pawar, V.M., Patel, S., Pathak, J., Swain, N., Hosalkar, R. and Iyer, J. (2015) Immunohistochemical Evaluation of Calretinin and Cytokeratin-19 in Odontogenic Keratocyst and Ameloblastoma: A Retrospective Study. Journal of Contemporary Dentistry, 5, 98-103. https://doi.org/10.5005/jp-journals-10031-1116

[28] Rudraraju, A., Venigalla, A., Babburi, S., Soujanya, P., Subramanyam, R.V. and Lakshmi, K.R. (2019) Calretinin Expression in Odontogenic Cystsand Odontogenic Tumors and the Possible Role of Calretinin in Pathogenesis of Ameloblastoma. Journal of Oral and Maxillofacial Pathology, 23, 349-355.

https://doi.org/10.4103/jomfp.JOMFP_54_19

[29] Kalsoom, F., Atique, M., Ahmed, S., Aslam, F., Sarfraz, T., Jamal, S., et al. (2015) Calretinin Expression in the Differential Diagnosis of Ameloblastoma and Keratocystic Odontogenic Tumour. Pakistan Armed Forces Medical Journal, 65, 8-12

[30] Imran, A., Ranganathan, K., Rao, U.D., Joshua, E. and Thavarajah, R. (2016) Expression of Calretinin and Cytokeratin 19 in Radicular Cyst, Dentigerous Cyst, Odontogenickerato Cyst, and Ameloblastoma. Journal of Dr. NTR University of Health Sciences, 5, 118. https://doi.org/10.4103/2277-8632.185442

[31] Coleman, H., Altini, M., Ali, H., Doglioni, C., Favia, G. and Maiorano, E. (2001) Use of Calretinin in the Differential Diagnosis of Unicystic Ameloblastomas. Histopathology, 38, 312-317. https://doi.org/10.1046/j.1365-2559.2001.01100.x

[32] Piattelli, A., Fioroni, M., Iezzi, G. and Rubini, C. (2003) Calretinin Expression in Odontogenic Cysts. Journal of Endodontics, 29, 394-396.

https://doi.org/10.1097/00004770-200306000-00003 